\title{
Intra-Cellular Therapies and Takeda to develop PDE1 inhibitors for schizophrenia
}

Takeda and Intra-Cellular Therapies, Inc. (ITI) have recently entered into an exclusive collaboration to discover, develop and commercialize phosphodiesterase type 1 (PDE1) inhibitors for treating cognitive impairment associated with schizophrenia a deal that could be worth US\$750 million for ITI.

"Cognitive impairment associated with schizophrenia is chronic, highly prevalent and not addressed by current therapies," says Paul Greengard, who is the Vincent Astor Professor at the Laboratory of Molecular and Cellular Neuroscience, The Rockefeller University, New York, USA, and co-founder and chairman of the scientific advisory board of ITI. All of these therapies are thought to act by modulating monoamine receptors (in particular the striatal dopamine $D_{2}$ receptor), and their limitations have stimulated interest in alternative strategies such as those targeting downstream intracellular signalling molecules.

PDE1 belongs to the PDE superfamily of enzymes, which degrade cyclic AMP and cyclic GMP — these are key second messenger molecules, and their intracellular concentrations are affected by modulation of $G$ protein-coupled receptors such as the dopamine $\mathrm{D}_{2}$ receptor. "In total, there are more than $100 \mathrm{PDE}$ isoforms, but selective PDE inhibitors could allow a more targeted approach [for treating schizophrenia] because of the specific localization of certain PDE isoforms in the brain," explains Jos Prickaerts, an associate professor at the Department of Psychiatry and Neuropsychology, Maastricht University, the Netherlands.

Indeed, a PDE10A inhibitor that is being developed by Pfizer (PF-2545920) for the treatment of schizophrenia is currently in Phase II trials. "PDE10A is expressed mostly in the striatum, but it is also expressed in the hippocampus and frontal cortex, and preclinical studies have shown that PDE10A inhibition has procognitive effects," says Prickaerts. "However, PDE1 is expressed mostly in the striatum as well, but it is more abundantly expressed than PDE10A in the hippocampus and frontal cortex, which suggests that PDE1 inhibition might have greater efficacy for cognitive enhancement than PDE10A inhibition." Greengard adds that "PDE1 is the only PDE that is calcium/ calmodulin-dependent. As such, PDE1 inhibitors have little or no effect on basal cyclic nucleotide levels; rather, they enhance cyclic nucleotide signalling in neurons under conditions that are associated with increased intracellular calcium levels. Other PDE inhibitors, including those directed at PDE10A, override the endogenous system by increasing the basal state of cyclic nucleotide signalling, which could be associated with unwanted side effects."

ITI currently have a series — named ITI-002 — of novel potent, orally available PDE1 inhibitors that are in preclinical development, although which of the three isoforms of PDE1 ( $A, B$ or $\mathrm{C}$ ) are being targeted has not been disclosed. This could be important for the biological effects sought, and represents a challenge in achieving the selectivity desired. "If PDE1B is the selective target, the effects will be more associated with striatal pathways, whereas if PDE1A is selectively targeted, the effects could differ substantially," says Akira Sawa, Director and Professor of the Program in Molecular Psychiatry at Johns Hopkins Hospital, Maryland, USA. "The distribution and role of PDE1A are thought to be more associated with the cerebral cortex than those of PDE1B, and the cortex is one of the most important brain areas involved in the pathophysiology of schizophrenia," he adds.

As well as potentially ameliorating the cognitive deficits in schizophrenia, PDE1 inhibitors might have utility in other neurological disorders. "PDE1 inhibitors may also be useful for treating cognitive impairment associated with Alzheimer's disease and Parkinson's disease. Moreover, they may also be useful as a form of adjunctive therapy to reduce the motor side effects of antipsychotic treatment in patients with schizophrenia," says Greengard. 